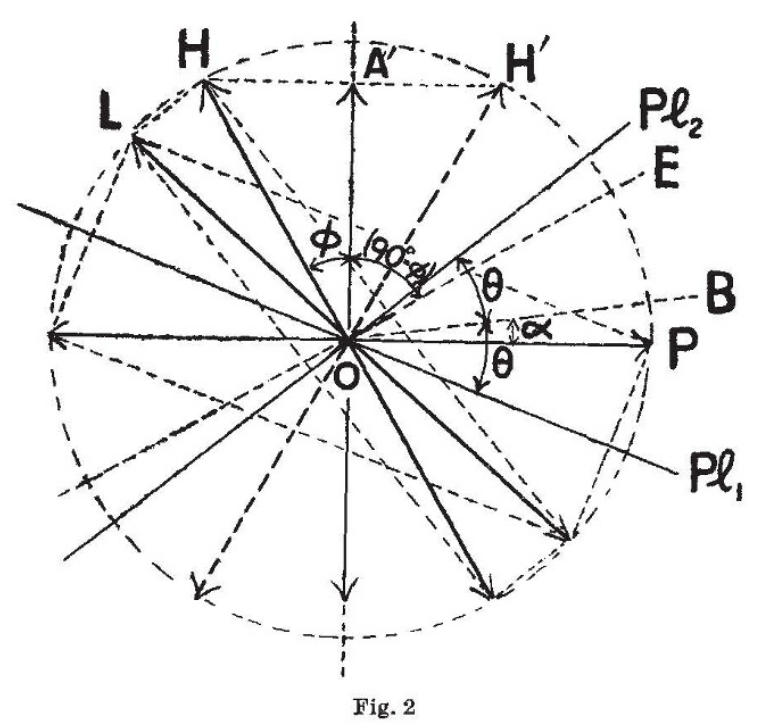

By means of equation (4) it should be possible to obtain at least approximate values of the spiral angle from measurements of the angle $(90-\varphi)$ made at the wave-length $\lambda_{180}$. This wave-length can be estimated roughly with the aid of suitable filters, or more accurately by means of a monochromator, as being that at which the closest agreement with the theoretical behaviour for the case $\delta=180^{\circ}$ is obtained.

This case and its consequences are not dealt with in any of the publications on fibre microscopy with which I am familiar.

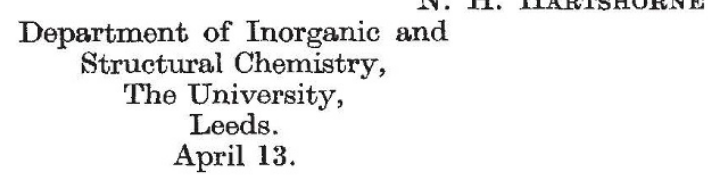

\section{Birefringence of Montmorillonite Complexes}

Doring a study of the optical properties of montmorillonite aggregates in salt solutions ${ }^{1}$, some interest. ing preliminary observations were made on various aromatic complexes. These observations have now been followed up with the view of extending the use of optical data in characterizing clays.

The structures of various aromatic complexes of montmorillonite have been inferred from spacing measurements and one-dimensional Fourier synthesis ${ }^{2}$. The monolayer complexes could be grouped into two classes, those where the plane of the ring was parallel to that of the silicate sheet (type $A$ ) and those where it was perpendicular (type $B$ ). Clearly, because the polarizability of the aromatic ring is usually much greater in the plane of the ring than perpendicular to $i^{3}$, the preferred orientation of the interlayer molecules in aromatic complexes should profoundly affect the apparent birefringence of the mineral. Thus, in type $B$ complexes, the effect of the interlayer molecules would be expected to reduce the strong negative birefringence of the silicate layers and even make some aggregates optically positive. In contrast, type $A$ complexes would be expected to be strongly negative. These expectations were strikingly confirmed when the birefringence of the nitrobenzene complex (type $B$ ) was found to be 0.003 (negative) as compared with 0.029 (negative) for the unexpanded mineral. An even larger effect was to be expected with quinoline, which is more anisotropic than nitrobenzene $\theta^{4}$, and the orientation of the interlayer molecules is such as to increase greatly the polarizability of the complex perpendicular to the silicate sheets ${ }^{4}$. Measurement of its birefringence showed it to be 0.02 (positive). In contrast, type $A$ complexes were found to be strongly negative, for example, pyridinium montmorillonite (type A) was 0.024 (negative).

These large birefringence changes obtained with montmorillonite not only confirm the proposed orientation of the interlayer molecules, but could be used to characterize clays in the same way as glycol and glycerol are used in X-ray diffraction analysis. For example, rapid assessment of elays for montmorillonite minerals is possible by measuring the changes in birefringence of aggregates on immersion in quinoline solutions. Where montmorillonite predominates, the optical sign is changed after treatment. Again, clays known to be homogeneous, but partially expanding and giving a complex X-ray diffraction pattern, have been rapidly and simply evaluated in terms of the percentage of expanding layers, from the change in birefringence in different solvents. When evaluation has been possible from $X$-ray results ${ }^{5}$, the agreement between the two methods has been excellent.

The preliminary part of this work was done while I held a Royal Society Exchange Fellowship, 1957-58, with the Academy of Sciences, U.S.S.R.

\section{R. GREENE-KELLY}

Rothamsted Experimental Station, Harpenden, Herts. May 12.

1 Derjaguin, B. V., and Greene-Kelly, R., (in the press). - Greene-Kelly, R., Trans. Farad. Soc., 51, 412 (1955)

'Stuart, H. A.. "Molekulstruktur" (Springer, Berlin, 1934).

- Le Fèvre, C. G., Le Fèvre, R. J. W. Purnachandra Rao, B., and Fmith, M. R., J. Chem. Soc., 1188 (1959).

${ }^{6}$ MaoEwan, D. M. C., Proc. Fourth U.S. Nat. Conf. on Clays and Clay Minerals $166(1956)$

\section{Electron Paramagnetic Resonance at $4 \cdot 2^{\circ} \mathrm{K}$. of $\lambda$-Irradiated Polymethyl Methacrylate and Polymethacrylic Acid}

THE electron paramagnetic resonance spectrum given by polymethyl methacrylate after high-energy irradiation at room temperature consists of five lines, about 23 gauss apart, with intensities in approximately the ratios $1: 4: 6: 4: 1$, with a weaker intermediate pattern of four lines, both closely centred on the electron spin $g$ factor of $2 \cdot 00^{1,2}$. Since this spectrum is also given by free radicals trapped during the addition polymerization of methyl methacrylate $^{3-5}$, it has been postulated that it arises from trapped propagating radicals of structure, $-\mathrm{CH}_{2}-$ $\dot{\mathrm{C}}\left(\mathrm{CH}_{3}\right) \mathrm{CO}_{2} \mathrm{CH}_{3}$, the detailed explanations involving the exact conformation of these radicals 2,4,6. Both of the explanations put forward by Symons ${ }^{4,6}$ require that the methyl group bonded to the 'radical' carbon atom rotates rapidly, so that its three protons interact equally with the unpaired electron. If one of these 